\title{
ERRATUM TO: A VARIATIONAL APPROACH TO GIVENTAL'S NONLINEAR MASLOV INDEX
}

\author{
Peter Albers and Urs Frauenfelder
}

\begin{abstract}
In this article we consider a variant of Rabinowitz Floer homology in order to define a homological count of discriminant points for paths of contactomorphisms. The growth rate of this count can be seen as an analogue of Givental's nonlinear Maslov index. As an application we prove a Bott-Samelson type obstruction theorem for positive loops of contactomorphisms.
\end{abstract}

\section{Introduction}

In [Giv89, Giv90a, Giv90b] Givental introduces his nonlinear Maslov index for the prequantization spaces $\mathbb{R} P^{2 n-1}$. This concept had remarkable applications to symplectic topology, for instance concerning the orderability of contact manifolds (see [EP00]) the existence of Calabi quasimorphisms (see [EP03,BS07]) and existence of Legendrian chords.

Let $(\Sigma, \xi=\operatorname{ker} \alpha)$ be a cooriented contact manifold. Givental's nonlinear Maslov index is formally defined as the intersection number of a path of contactomorphisms with the discriminant

$$
\left\{\varphi \in \operatorname{Cont}(\Sigma, \xi) \mid \exists x \in \Sigma \text { such that } \varphi(x)=x \text { and }\left.\varphi^{*} \alpha\right|_{x}=\left.\alpha\right|_{x}\right\} .
$$

Unfortunately, the discriminant has codimension-1 singularities (see [Giv90b]). For $\Sigma=\mathbb{R P}^{2 n-1}$ Givental resolves this problem by constructing the tail or train which is a subset of the discriminant and defines an cooriented codimension-1 cycle. The nonlinear Maslov index on $\mathbb{R P}^{2 n-1}$ is then the intersection number with this cycle. It seems very difficult to extend Givental's constructions to other contact manifolds. Givental already suggested to use Floer theoretic methods in the general case. In this article we define a homological count of discriminant points for positive paths of contactomorphisms (see Definition 2.1). Positive paths play a prominent role in the theory of orderable contact manifolds by Eliashberg and Polterovich [EP00]. We

The online version of the original article can be found under doi:10.1007/s00039-012-0187-2.

Due to an unfortunate error during the processing of the article by the publisher, the spelling of the second author's name was incorrect. The name should read Urs Frauenfelder.

Keywords and phrases: Nonlinear Maslov index, discriminant points, Rabinowitz Floer homology 
use the following observation to give a variational characterization of the discriminant.

OBSERVATION (cf. Remark 3.3). For every positive path of contactomorphisms there exists a time-dependent Rabinowitz action functional whose critical points are in 1-1 correspondence to the discriminant points of the positive path.

Theorem (cf. Theorems 4.8 and 4.13 and Definition 4.16). If the contact manifold admits an exact symplectic filling then we can assign a filtered Rabinowitz Floer homology group to a generic positive path of contactomorphisms. It depends only on the homotopy class with fixed end points of the positive path and is invariant under conjugation of the path.

Our replacement for Givental's nonlinear Maslov index for a positive path is the growth rate of the filtered Rabinowitz Floer homology groups associated to this positive path. We point out that the Rabinowitz Floer homology groups depend on the choice of an exact symplectic filling as explicit examples show (see Section 5). For unit cotangent bundles the growth rate of the Rabinowitz Floer homology groups is related to growth rates of geodesics. As an application we prove the following theorem.

Theorem (cf. Theorem 7.1). Let $B$ be a closed manifold with finite fundamental group such that the rational cohomology ring has at least two generators. Then $\Sigma:=S^{*} B$ with its standard contact structure $\xi$ admits no closed positive loops in $\operatorname{Cont}(\Sigma, \xi)$.

This can be thought of as a generalization of the classical Bott-Samelson theorem (see [Bes78]) to positive loops of contactomorphisms.

REMARK 1.1. Eliashberg and Polterovich [EP00] that the absence of positive contractible loops of contactomorphisms implies that the cone in $\widetilde{\operatorname{Cont}_{0}}(\Sigma, \xi)$ given by non-negative paths of contactomorphisms defines a partial order on $\widetilde{\operatorname{Cont}_{0}}(\Sigma, \xi)$.

Leonid Polterovich explained to us that the absence of positive loops (contractible or not) implies that the cone in $\operatorname{Cont}_{0}(\Sigma, \xi)$ given by contactomorphisms which can be joined to the identity by a non-negative path defines a partial order on $\operatorname{Cont}_{0}(\Sigma, \xi)$. In other words the partial order descends from the universal cover $\widetilde{\operatorname{Cont}_{0}}(\Sigma, \xi)$ to $\operatorname{Cont}_{0}(\Sigma, \xi)$.

Finally we like to point out that in Section 5 we show that the homological intersection number defined below detects the rotation number for $\Sigma=S^{1}$. On $S^{1}$ Givental's nonlinear Maslov index is given by the rotation number.

\section{Positive Contact Isotopies}

Let $(\Sigma, \xi)$ be a cooriented contact manifold. We fix a contact form $\alpha$ for $\xi$. 
Definition 2.1. A smooth path $\left\{\varphi_{t}\right\}_{t \in \mathbb{R}}$ in $\operatorname{Cont}(\Sigma)$ based at the identity is called positive resp. twisted periodic if the function $h_{t}: \Sigma \rightarrow \mathbb{R}$ defined by

$$
h_{t}\left(\varphi_{t}(x)\right):=\alpha_{\varphi_{t}(x)}\left(\frac{d}{d t} \varphi_{t}(x)\right)
$$

is positive resp. 1-periodic. We set

$$
\mathcal{P} \equiv \mathcal{P}(\Sigma, \xi):=\left\{\left\{\varphi_{t}\right\}_{t \in \mathbb{R}} \mid\left\{\varphi_{t}\right\}_{t \in \mathbb{R}} \text { is positive and twisted periodic }\right\} .
$$

REMARK 2.2. The above definition is independent of the chosen contact form as long as it defines the same coorientation. Moreover, $\varphi_{t}$ is twisted periodic if and only if $\varphi_{t+1}=\varphi_{t} \varphi_{1}$ for all $t \in \mathbb{R}$. In particular, a twisted periodic path satisfies $\varphi_{1}^{m}=\varphi_{m}$ for all $m \in \mathbb{Z}$.

We denote by $\left(S \Sigma:=\Sigma \times \mathbb{R}_{>0}, \omega:=d(r \alpha)\right), r \in \mathbb{R}_{\geq 0}$, the symplectization of $\Sigma$.

Proposition 2.3. The contact isotopy $\varphi_{t}$ admits a lift to a Hamiltonian isotopy $\phi_{t}$ of $S \Sigma$ as follows:

$$
\phi_{t}(x, r):=\left(\varphi_{t}(x), \frac{r}{\rho_{t}(x)}\right): S \Sigma \rightarrow S \Sigma
$$

where $\rho_{t}(x): \Sigma \rightarrow \mathbb{R}_{>0}$ is defined by $\left.\varphi_{t}^{*} \alpha\right|_{x}=\left.\rho_{t}(x) \alpha\right|_{x}$. Moreover, $\phi_{t}$ is generated by the Hamiltonian function $H_{t}: S \Sigma \rightarrow \mathbb{R}$ given by

$$
H_{t}(x, r)=r h_{t}(x) \text {. }
$$

The proof of Proposition 2.3 can be found after Remark 2.6.

Definition 2.4. The function $H_{t}: S \Sigma \rightarrow \mathbb{R}$ is called the contact Hamiltonian associated to $\left\{\varphi_{t}\right\}$.

Following Givental [Giv89, Giv90a, Giv90b] we make the following definition.

Definition 2.5. Let $\left\{\varphi_{t}\right\}$ be a smooth path in $\operatorname{Cont}(\Sigma)$. Then a pair $(x, \eta) \in \Sigma \times \mathbb{R}$ is called a discriminant point (with respect to $\left\{\varphi_{t}\right\}$ ) if

$$
\left\{\begin{array}{l}
\varphi_{\eta}(x)=x \\
\left.\varphi_{\eta}^{*} \alpha\right|_{x}=\left.\alpha\right|_{x}
\end{array}\right.
$$

REMARK 2.6. We point out that for a pair $(x, \eta)$ being a discriminant point is equivalent to $\phi_{\eta}(x, r)=(x, r)$ for any $r>0$ (see Proposition 2.3$)$.

Proof of Proposition 2.3. We prove the stronger fact that $\phi_{t}$ preserves the 1-form $r \alpha$ :

$$
\left.\phi_{t}^{*}(r \alpha)\right|_{(x, r)}=\left.\frac{r}{\rho_{t}(x)} \cdot \varphi_{t}^{*} \alpha\right|_{x}=\left.r \alpha\right|_{x}
$$


We set

$$
Y_{t}\left(\varphi_{t}(x)\right):=\frac{d}{d t} \varphi_{t}(x)
$$

and compute

$$
\begin{aligned}
X_{t}\left(\phi_{t}(x, r)\right) & :=\frac{d}{d t} \phi_{t}(x, r) \\
& =Y_{t}\left(\varphi_{t}(x)\right)-r \frac{\dot{\rho}_{t}(x)}{\rho_{t}^{2}(x)} \frac{\partial}{\partial r} .
\end{aligned}
$$

Since $\phi_{t}$ preserves $\lambda:=r \alpha$ we use Lemma 2.7 and compute

$$
H_{t}=\lambda\left(X_{t}\right)=r \alpha\left(Y_{t}\right)=r h_{t} .
$$

Lemma 2.7. Let $\omega=d \lambda$ be an exact symplectic form and $X$ a vector field satisfying

$$
\mathcal{L}_{X} \lambda=0
$$

where $\mathcal{L}$ is the Lie derivative. Then the Hamiltonian vector field $X_{H}$ of the function $H:=\lambda(X)$ equals $X$ :

$$
X_{H}=X
$$

Proof. From $H=i_{X} \lambda$ we compute using Cartan's formula

$$
\begin{aligned}
d H & =d\left(i_{X} \lambda\right) \\
& =7 \mathcal{L}_{X} \lambda-i_{X} d \lambda \\
& =-i_{X} \omega .
\end{aligned}
$$

REMARK 2.8. In particular, we have the equality

$$
\lambda\left(X_{H}\right)=H
$$

\section{The Rabinowitz Action Functional for Time-dependent} Hamiltonians and a Variational Approach to Discriminant Points

Let $(M, \omega=d \lambda)$ be an exact symplectic manifold and $F: M \times \mathbb{R} \rightarrow \mathbb{R}$ a smooth function. We denote by $\mathscr{L}:=W^{1,2}(\mathbb{R} / \mathbb{Z}, M)$ the free loop space of $M$ and define the Rabinowitz action functional

$$
\begin{aligned}
\mathcal{A}: \mathscr{L} \times \mathbb{R} \longrightarrow \mathbb{R} & \\
(u, \eta) & \mapsto \mathcal{A}(u, \eta)=\int_{0}^{1} u^{*} \lambda-\eta \int_{0}^{1} F_{\eta t}(u(t)) d t .
\end{aligned}
$$


Its critical points $(u, \eta) \in \operatorname{Crit} \mathcal{A}$ satisfy

$$
\left.\begin{array}{l}
\dot{u}(t)=\eta X_{F_{\eta t}}(u(t)) \\
\int_{0}^{1}\left[F_{\eta t}(u(t))+\eta t \dot{F}_{\eta t}(u(t))\right] d t=0
\end{array}\right\} .
$$

By the first equation we have

$$
\begin{aligned}
\frac{d}{d t} F_{\eta t}(u(t)) & =\eta \dot{F}_{\eta t}(u(t))+d F_{\eta t}(u(t))[\dot{u}(t)] \\
& =\eta \dot{F}_{\eta t}(u(t))+d F_{\eta t}(u(t))\left[\eta X_{F_{\eta t}}(u(t))\right] \\
& =\eta \dot{F}_{\eta t}(u(t))-\underbrace{\omega\left(X_{F_{\eta t}}(u(t)), \eta X_{F_{\eta t}}(u(t))\right)}_{=0} .
\end{aligned}
$$

Thus, the second equation becomes after integration by parts

$$
\begin{aligned}
0 & =\int_{0}^{1}\left[F_{\eta t}(u(t))+\eta t \dot{F}_{\eta t}(u(t))\right] d t \\
& =\int_{0}^{1}\left[F_{\eta t}(u(t))+t \frac{d}{d t} F_{\eta t}(u(t))\right] d t \\
& =\int_{0}^{1}[\underbrace{F_{\eta t}(u(t))-\left(\frac{d}{d t} t\right) F_{\eta t}(u(t))}_{=0}] d t+\left.t F_{\eta t}(u(t))\right|_{0} ^{1} \\
& =F_{\eta}(u(1)) .
\end{aligned}
$$

We proved the following lemma.

Lemma 3.1. A pair $(u, \eta) \in \mathscr{L} \times \mathbb{R}$ is a critical point of $\mathcal{A}$ if and only if the following equations hold

$$
\left.\begin{array}{l}
\dot{u}(t)=\eta X_{F_{\eta t}}(u(t)) \\
F_{\eta}(u(1))=0
\end{array}\right\} .
$$

LEMMA 3.2. If the function $F_{t}$ satisfies

$$
\lambda\left(X_{F_{t}}\right)=F_{t}+\kappa
$$

for some $\kappa \in \mathbb{R}$ then

$$
\mathcal{A}(u, \eta)=\kappa \eta \quad \forall(u, \eta) \in \operatorname{Crit} \mathcal{A}
$$


Proof. Using the critical point equation for $\mathcal{A}$ we see

$$
\begin{aligned}
\mathcal{A}(u, \eta) & =\int_{0}^{1} \lambda\left[\eta X_{F_{\eta t}}(u(t))\right] d t-\eta \int_{0}^{1} F_{\eta t}(u(t)) d t \\
& =\int_{0}^{1} \eta F_{\eta t}(u(t)) d t+\kappa \eta-\eta \int_{0}^{1} F_{\eta t}(u(t)) d t \\
& =\kappa \eta .
\end{aligned}
$$

REMARK 3.3. If $F_{t}=r h_{t}(x)-\kappa$, where $r h_{t}$ is the contact Hamiltonian of a positive and twisted periodic path $\left\{\varphi_{t}\right\} \in \mathcal{P}$, then discriminant points are in 1-1 correspondence with critical points of $\mathcal{A}_{\kappa}:=\frac{1}{\kappa} \mathcal{A}$ where $M=S \Sigma$ (see Proposition 2.3 and Lemma 3.1).

\section{A Homological Maslov Index (Periodic Case)}

Let $(\Sigma, \xi)$ be a closed, cooriented contact manifold and $\alpha$ a fixed contact form. We assume that there exists a compact exact symplectic manifold $(\widetilde{M}, d \widetilde{\lambda})$ with $\Sigma=\partial \widetilde{M}$ and $\alpha=\left.\lambda\right|_{\Sigma}$. We attach to $\widetilde{M}$ the positive part of the symplectization of $\Sigma$, that is,

$$
M:=\widetilde{M} \cup_{\Sigma} \Sigma \times\{r \geq 1\} .
$$

On $M$ we define a 1 -form $\lambda$ by $\widetilde{\lambda}$ on $\widetilde{M}$ and $\lambda=r \alpha$ on $\Sigma \times\{r \geq 1\}$. In particular, $(M, \omega=d \lambda)$ is an exact symplectic manifold. We point out, that the entire symplectization $S \Sigma$ of $\Sigma$ embeds into $M$ via the flow of the Liouville vector field of $\lambda$.

Convention 4.1. In the following we only consider positive and twisted periodic path, i.e. $\left\{\varphi_{t}\right\} \in \mathcal{P}(\Sigma, \xi)$ (see Definition 2.1).

We need to extend the functions of the form $F_{t}=r h_{t}(x)-\kappa$ defined on the symplectization $S \Sigma$ over the filling $\widetilde{M}$. In order to control the actions of additional critical points of $\mathcal{A}$ coming from the extension of $F_{t}$ we fix $R, \kappa>1$ with $R \kappa>2$ and choose a smooth function $\beta_{R}: \mathbb{R}_{\geq 0} \rightarrow[0,1]$ satisfying

$$
\beta_{R}(r)= \begin{cases}0 & r \leq 1 \\ 1 & 2 \leq r \leq R \kappa \\ 0 & r \geq R \kappa+1\end{cases}
$$

and

$$
\begin{cases}0 \leq \beta_{R}^{\prime}(r) \leq 2 & 1 \leq r \leq 2 \\ -2 \leq \beta_{R}^{\prime}(r) \leq 0 & R \kappa \leq r \leq R \kappa+1\end{cases}
$$


Moreover, we define

$$
\mathfrak{h}(r)= \begin{cases}m & r \leq 2 \\ M & r>2\end{cases}
$$

where

$$
0<m \leq \min \left\{h_{t}(x) \mid x \in \Sigma, t \in \mathbb{R}\right\}
$$

and

$$
M \geq \max \left\{h_{t}(x) \mid x \in \Sigma, t \in \mathbb{R}\right\} .
$$

$m$ and $M$ are well-defined since $h_{t}$ is 1 -periodic. We set

$$
F_{t}^{\kappa, R}(z):= \begin{cases}r\left[\beta_{R}(r) h_{t}(x)+\left(1-\beta_{R}(r)\right) \mathfrak{h}(r)\right]-\kappa & z=(x, r) \in S \Sigma \\ -\kappa & z \in M \backslash S \Sigma\end{cases}
$$

and consider the normalized Rabinowitz action functional

$$
\begin{aligned}
& \mathcal{A}_{\kappa, R}: \mathscr{L} \times \mathbb{R} \longrightarrow \mathbb{R} \\
& \quad(u, \eta) \mapsto \mathcal{A}_{\kappa, R}(u, \eta)=\frac{1}{\kappa}\left(\int_{0}^{1} u^{*} \lambda-\eta \int_{0}^{1} F_{\eta t}^{\kappa, R}(u(t)) d t\right) .
\end{aligned}
$$

Obviously, the critical point equation does not change if we divide by $\kappa$, thus $(u, \eta) \in$ $\operatorname{Crit} \mathcal{A}_{\kappa, R}$ if and only if

$$
\left.\begin{array}{l}
\dot{u}(t)=\eta X_{F_{\eta t}^{\kappa, R}}(u(t)) \\
F_{\eta}^{\kappa, R}(u(1))=0
\end{array}\right\}
$$

A glimpse at Lemma 3.2 reveals the reason why we divide by $\kappa$. In order to define Floer homology for $\mathcal{A}_{\kappa, R}$ it is necessary to establish a bound on the Lagrange multiplier $\eta$ along gradient flow lines in terms of the action of the corresponding critical points (see Lemma 4.5). For this we need the following.

Lemma 4.2. Let $(u, \eta) \in \operatorname{Crit} \mathcal{A}_{\kappa, R}$ be a critical point. Then

$$
\left|\mathcal{A}_{\kappa, R}(u, \eta)\right| \geq|\eta| .
$$

Proof. We compute

$$
\begin{aligned}
\lambda\left(X_{F_{\eta t}^{\kappa, R}}\right) & =d F_{\eta t}^{\kappa, R}\left(r \frac{\partial}{\partial r}\right) \\
& =F_{\eta t}^{\kappa, R}+\kappa+r^{2} \beta_{R}^{\prime}(r)\left[h_{\eta t}(x)-\mathfrak{h}(r)\right] .
\end{aligned}
$$

We point out that

$$
\beta_{R}^{\prime}(r)\left[h_{\eta t}(x)-\mathfrak{h}(r)\right] \geq 0
$$


holds (see (4.2) and (4.3)). We estimate

$$
\begin{aligned}
\left|\mathcal{A}_{\kappa, R}(u, \eta)\right| & =\frac{1}{\kappa}\left|\int_{0}^{1} \lambda\left(\eta X_{F_{\eta t}^{\kappa, R}}(u)\right)-\eta \int_{0}^{1} F_{\eta t}^{\kappa, R}(u) d t\right| \\
& =\frac{|\eta|}{\kappa}\left|\int_{0}^{1}\left[F_{\eta t}^{\kappa, R}(u)+\kappa+r^{2} \beta_{R}^{\prime}(r)\left[h_{\eta t}(x)-\mathfrak{h}(r)\right]-F_{\eta t}^{\kappa, R}(u)\right] d t\right| \\
& =\frac{|\eta|}{\kappa}|\kappa+\int_{0}^{1} r^{2} \underbrace{\beta_{R}^{\prime}(r)\left[h_{\eta t}(x)-\mathfrak{h}(r)\right]}_{\geq 0} d t| \\
& \geq|\eta| .
\end{aligned}
$$

This finishes the proof.

In the next proposition and corollary we prove that in a given action window no additional critical points appear for appropriate choices of $\kappa$ and $R$.

Proposition 4.3. Given $a<b$ there exists $\kappa_{0}=\kappa_{0}(a, b)>0$ and $R_{0}=R_{0}(a, b) \geq 0$ such that for all $\kappa \geq \kappa_{0}$ and $R \geq R_{0}$ the following holds. Let $(u, \eta) \in \operatorname{Crit} \mathcal{A}_{\kappa, R}$ be a critical point with critical value between $a$ and $b$

$$
a<\mathcal{A}_{\kappa, R}(u, \eta)<b
$$

then $u(t)=(x(t), r(t)) \in \Sigma \times(2, R \kappa)$ for all $t \in S^{1}$ and $\mathcal{A}_{\kappa, R}(u, \eta)=\eta$.

Proof. By Lemma 4.2 we have

$$
|\eta| \leq \max \{|a|,|b|\}
$$

We set

$$
C \equiv C(a, b):=\max \left\{\left|\eta \cdot \frac{\dot{\rho}_{\eta t}(x)}{\rho_{\eta t}^{2}(x)}\right|: x \in \Sigma, t \in[0,1],|\eta| \leq \max \{|a|,|b|\}\right\} .
$$

We fix $\kappa_{0}>\max \left\{1,3 M e^{C}\right\}, R_{0}>\max \left\{\frac{1}{m} e^{C}+1, \frac{1}{M}\right\}$ and choose $\kappa \geq \kappa_{0}$ and $R \geq R_{0}$.

Step 1: We prove that $u(1)=(x(1), r(1)) \in \Sigma \times[2, R \kappa]$ and $r(1) h_{\eta}(x(1))=\kappa$.

Proof of Step 1. We examine three cases.

Case 1: We assume that $u(1) \notin \Sigma \times[1, R \kappa+1]$.

We first observe that if $u(1) \notin S \Sigma$ then $F_{\eta}^{\kappa, R}(u(1))=-\kappa<0$. Therefore, the critical point equation implies $u(1)=(x(1), r(1)) \in S \Sigma$. Since $r(1) \notin[1, R \kappa+1]$ we have $\beta_{R}(r(1))=0$ and therefore

$$
0=F_{\eta}^{\kappa, R}(u(1))=r(1) \mathfrak{h}(r(1))-\kappa .
$$


So either $r(1) \leq 1$ and $\kappa=r(1) m \leq m$ or $r(1) \geq R \kappa+1$ and $\kappa=r(1) M \geq R M \kappa+M$. The former contradicts the assumption $\kappa \geq \kappa_{0}>3 M e^{C}>m$ and the latter contradicts the assumption $R M>1$.

Case 2: We assume that $1 \leq r(1) \leq 2$.

For simplicity we write $r=r(1)$ and $x=x(1)$. Using $M \geq h_{\eta}(x) \geq \mathfrak{h}(r)=m$ and $0 \leq \beta_{R}(r) \leq 1$ we estimate using the critical point equation

$$
\begin{aligned}
\kappa & =r\left[\beta_{R}(r) h_{\eta}(x)+\left(1-\beta_{R}(r)\right) \mathfrak{h}(r)\right] \\
& =r\left[\beta_{R}(r)\left(h_{\eta}(x)-\mathfrak{h}(r)\right)+\mathfrak{h}(r)\right] \\
& \leq r\left[\left(h_{\eta}(x)-\mathfrak{h}(r)\right)+\mathfrak{h}(r)\right] \\
& \leq r h_{\eta}(x) \\
& \leq r M \\
& \leq 2 M .
\end{aligned}
$$

This contradicts $\kappa_{0}>3 M e^{C}>2 M$.

Case 3: We assume that $R \kappa \leq r(1) \leq R \kappa+1$.

Again for simplicity we write $r=r(1)$ and $x=x(1)$. Using that $h_{\eta}(x) \leq \mathfrak{h}(r)$ and $\beta(r) \geq 0$ we estimate

$$
\begin{aligned}
\kappa & =r\left[\beta_{R}(r)\left(h_{\eta}(x)-\mathfrak{h}(r)\right)+\mathfrak{h}(r)\right] \\
& \geq r\left[\left(h_{\eta}(x)-\mathfrak{h}(r)\right)+\mathfrak{h}(r)\right] \\
& =r h_{\eta}(x) \\
& \geq R \kappa m .
\end{aligned}
$$

This contradicts the assumption $R M \geq R m>1$.

From the three cases we conclude that $2 \leq r(1) \leq R \kappa$. The definition of $\beta_{R}$ and the critical point equation (see Lemma 3.1) imply

$$
0=F_{\eta}^{\kappa, R}(u(1))=r(1) h_{\eta}(x(1))-\kappa .
$$

This proves Step 1.

Step 2: We prove that $u(t)=(x(t), r(t)) \in \Sigma \times(2, R \kappa)$ for all $t \in S^{1}$.

Proof of Step 2. We set

$$
I:=\{t \in[0,1] \mid u(t) \in \Sigma \times(2, R \kappa)\}
$$

By Step 1 we have

$$
\frac{\kappa}{M} \leq r(0)=r(1)=\frac{\kappa}{h_{\eta}(x(1))} \leq \frac{\kappa}{m} .
$$

Then since $R \geq R_{0} \geq \frac{1}{m} e^{C}+1 \geq \frac{1}{m}+1$ and $\kappa \geq \kappa_{0} \geq 1$ we see

$$
\frac{\kappa}{m} \leq(R-1) \kappa \leq R \kappa-1 \text {. }
$$


Moreover, since $\kappa \geq \kappa_{0} \geq 3 M e^{C} \geq 3 M$ we have

$$
3 \leq r(0)=r(1) \leq R \kappa-1 .
$$

Thus, $0 \in I \neq \emptyset$. We denote by $I_{0}$ the connected component of $I$ containing 0 .

Claim. If $t \in I_{0}$ then $3 \leq r(t) \leq R \kappa-1$.

Proof of the Claim. As long as $u(t)=(x(t), r(t)) \in \Sigma \times[2, R \kappa]$ the function $r(t)$ satisfies

$$
\dot{r}(t)=-\eta r(t) \frac{\dot{\rho}_{\eta t}(x(t))}{\rho_{\eta t}^{2}(x(t))},
$$

see (2.8) together with the critical point equation. Thus, for $t \in I_{0}$ we can estimate

$$
r(0) e^{-C} \leq r(t) \leq r(0) e^{C}
$$

where $C \equiv C(a, b)$ is defined in (4.16). By Step 1 we have $\frac{\kappa}{M} \leq r(0)=r(1) \leq \frac{\kappa}{m}$ and we obtain

$$
\frac{\kappa}{M} e^{-C} \leq r(t) \leq \frac{\kappa}{m} e^{C}
$$

Since $\kappa \geq \kappa_{0} \geq 3 M e^{C}$ we see

$$
r(t) \geq 3
$$

Since $R \geq R_{0} \geq \frac{1}{m} e^{C}+1$ and $\kappa \geq \kappa_{0} \geq 1$ we have

$$
\begin{aligned}
r(t) & \leq \kappa \frac{1}{m} e^{C} \\
& \leq \kappa(R-1) \\
& \leq \kappa R-1 .
\end{aligned}
$$

This proves the Claim.

By definition $I_{0}$ is open. By the Claim it is also closed. Since $I_{0} \neq \emptyset$ we conclude $I_{0}=I=[0,1]$. This proves Step 2 .

Since on $\Sigma \times(2, R \kappa-1)$ we have $F_{t}^{\kappa, R}(u)=r h_{t}(x)-\kappa$. Thus, we get

$$
\lambda\left(X_{F_{t}^{\kappa, R}}\right)=F_{t}^{\kappa, R}+\kappa .
$$

Therefore, Lemma 3.2 implies

$$
\mathcal{A}_{\kappa, R}(u, \eta)=\eta
$$

for all critical points contained in $\Sigma \times(2, R \kappa-1)$. This finishes the proof of Proposition 4.3. 
Corollary 4.4. We fix $a<b$. If $\kappa \geq \kappa_{0}$ and $R \geq R_{0}$ where $\kappa_{0}$ and $R_{0}$ are the constants in Proposition 4.3 then the critical point equation and the critical value for critical points of $\mathcal{A}_{\kappa, R}$ with action values $a<\mathcal{A}_{\kappa, R}<b$ are independent of $\kappa$ and $R$. Moreover, they are critical points of $\mathcal{A}$ and thus correspond to discriminant points (see Remark 3.3).

Proof. From Proposition 4.3 we know that critical points with action values $a<$ $\mathcal{A}_{\kappa, R}<b$ are contained in $\Sigma \times(2, R \kappa-1)$. On $\Sigma \times(2, R \kappa-1)$ we have $F_{t}^{\kappa, R}(u)=$ $r h_{t}(x)-\kappa$. Thus, $F_{t}^{\kappa, R}(u)$ is independent of $R$. Therefore, the critical point equation is independent (up to a $\kappa$-shift in the $r$-direction of the symplectization). The critical value is independent of $\kappa$ due to the normalization (see (4.8)). Remark 3.3 implies the statement about critical points of $\mathcal{A}$ and discriminant points.

Now we are in the position to construct Floer homology for $\mathcal{A}_{\kappa, R}$. We choose an almost complex structure $J$ which on $\Sigma \times[1, \infty)$ is of SFT-type (see [CFO10]). We define for $\kappa>0$ the $L^{2}$-metric $\mathfrak{m}^{\kappa}$ on $\mathscr{L} \times \mathbb{R}$ by

$$
\mathfrak{m}_{(z, \eta)}^{\kappa}\left((\xi, l),\left(\xi^{\prime}, l^{\prime}\right)\right):=\frac{1}{\kappa} \int_{0}^{1} \omega_{(z, \eta)}\left(\xi, J \xi^{\prime}\right) d t+\frac{l l^{\prime}}{\kappa} .
$$

Then the gradient of $\mathcal{A}_{\kappa, R}$ at $(u, \eta) \in \mathscr{L} \times \mathbb{R}$ equals

$$
\nabla^{\kappa} \mathcal{A}_{\kappa, R}(u, \eta)=\left(\begin{array}{c}
\dot{u}(t)-\eta X_{F_{\eta t}^{\kappa, R}}(u(t)) \\
\int_{0}^{1}\left[F_{\eta t}^{\kappa, R}(u(t))+\eta t \dot{F}_{\eta t}^{\kappa, R}(u(t))\right] d t
\end{array}\right)
$$

and its norm

$$
\begin{aligned}
\left\|\nabla^{\kappa} \mathcal{A}_{\kappa, R}(u, \eta)\right\|_{\kappa}^{2}= & \frac{1}{\kappa}\left\|\dot{u}(t)-\eta X_{F_{\eta t}^{\kappa, R}}(u(t))\right\|_{2}^{2} \\
& +\frac{1}{\kappa}\left(\int_{0}^{1}\left[F_{\eta t}^{\kappa, R}(u(t))+\eta t \dot{F}_{\eta t}^{\kappa, R}(u(t))\right] d t\right)^{2} .
\end{aligned}
$$

Lemma 4.2 asserts that at critical points the Lagrange multiplier $\eta$ is bounded by the action. This continues to hold for almost critical points.

Lemma 4.5 (Fundamental lemma). There exists $\epsilon>0$ such that for all $w=(u, \eta) \epsilon$ $\mathscr{L} \times \mathbb{R}$ we have

$$
\left\|\nabla^{\kappa} \mathcal{A}_{\kappa, R}(w)\right\|_{\kappa}<\epsilon \Longrightarrow|\eta| \leq \frac{1}{\epsilon}\left(\mathcal{A}_{\kappa, R}(w)+1\right) .
$$

Proof. The proof follows by a standard scheme (see [CF09]). 
We point out that for $r$ sufficiently large the Hamiltonian function equals $F_{t}^{\kappa, R}(x, r)=M r-\kappa$. Thus, we can apply the techniques from [CFO10] to obtain $L^{\infty}$-bounds for the $r$-coordinate of solutions of the Rabinowitz-Floer equation. $L^{\infty}$ bounds for the Lagrange multiplier follow again by a standard scheme from the Fundamental Lemma 4.5. Finally, there is no bubbling-off of holomorphic spheres since the symplectic manifold $M$ was assumed to be exact.

We recall (see Remark 3.3) that a positive and twisted periodic path $\left\{\varphi_{t}\right\}$ of contactomorphisms defines a Rabinowitz action functional $\mathcal{A}$ whose critical points are in 1-1 correspondence with discriminant points. Moreover, the choice of $\kappa_{0}$ and $R_{0}$ guarantee that the critical points of $\mathcal{A}_{\kappa, R}$ are exactly the critical points of $\mathcal{A}$.

Definition 4.6. We call a path $\left\{\varphi_{t}\right\} \in \mathcal{P}$ non-degenerate if the Rabinowitz action functional $\mathcal{A}_{\kappa}=\frac{1}{\kappa} \mathcal{A}$ is Morse for one (and then any) $\kappa$.

REMARK 4.7. Since positive and twisted periodic paths are generated by timedependent, 1-periodic functions it is straight forward to see that they are generically non-degenerate.

Theorem 4.8. Let $\left\{\varphi_{t}\right\}$ be non-degenerate. Then for $a<b$ and $\kappa \geq \kappa_{0}(a, b), R \geq$ $R_{0}(a, b)$ Rabinowitz Floer homology $\operatorname{RFH}_{a}^{b}\left(\mathcal{A}_{\kappa, R}\right)$ is well-defined and independent of $\kappa$ and $R$ up to chain complex isomorphisms. For simplicity we use $\mathbb{Z} / 2$-coefficients.

Proof. By the previous remarks compactness up to breaking of gradient flow lines (in the sense of Floer) is guaranteed. Thus, $\operatorname{RFH}_{a}^{b}\left(\mathcal{A}_{\kappa, R}\right)$ is defined.

Since the critical points and values are independent of $\kappa$ and $R$ a continuation argument implies that $\operatorname{RFH}_{a}^{b}\left(\mathcal{A}_{\kappa, R}\right)$ is independent of $\kappa$ and $R$ up to chain complex isomorphisms.

Definition 4.9. Let $\left\{\varphi_{t}\right\}$ be non-degenerate. Then we define the filtered Rabinowitz Floer homology of $\left\{\varphi_{t}\right\}$ to be

$$
\operatorname{RFH}_{a}^{b}\left(\left\{\varphi_{t}\right\}\right):=\operatorname{RFH}_{a}^{b}\left(\mathcal{A}_{\kappa, R}\right)
$$

for some $\kappa \geq \kappa_{0}(a, b), R \geq R_{0}(a, b)$.

REMARK 4.10. We point out that $\operatorname{RFH}_{a}^{b}\left(\left\{\varphi_{t}\right\}\right)$ possibly depends on the filling $\widetilde{M}$ of $\Sigma$ (see Section 5$)$. Nevertheless, we suppress this in the notation.

Definition 4.11. A path $\left\{\varphi_{t}\right\} \in \mathcal{P}$ is non-resonant if $\mathcal{A}_{\kappa}$ has no integer critical values for one (and then any) $\kappa>0$ (see Remark 3.3). Then for $n, m \in \mathbb{Z}$ we define

$$
\operatorname{RFH}_{n}^{m}\left(\left\{\varphi_{t}\right\}\right)
$$

using a sufficiently small perturbation of $\left\{\varphi_{t}\right\}$ which is non-degenerate. 
REMARK 4.12. $\operatorname{RFH}_{n}^{m}\left(\left\{\varphi_{t}\right\}\right)$ is well-defined for non-resonant $\left\{\varphi_{t}\right\}$ since any sufficiently small perturbation is non-resonant and non-degenerate. Moreover, during a sufficiently small perturbation no critical values crosses an integer.

Moreover, a path $\left\{\varphi_{t}\right\} \in \mathcal{P}$ being non-resonant is a property depending only of $\varphi_{1}$. Indeed, the critical value of $(u, \eta)$ equals $\eta$ and thus, if $\eta \in \mathbb{Z}$ then $\varphi_{\eta}=\varphi_{1}^{\eta}$. In particular, the discriminant points $(x, \eta)$ with $\eta \in \mathbb{Z}$ depend only on $\varphi_{1}$.

The same reasoning implies the following theorem.

Theorem 4.13. Let $I \subset \mathbb{R}$ be some interval and $\left\{\varphi_{t, s}\right\}_{t \in \mathbb{R}, s \in I}$ be a smooth family of contactomorphisms such that for all fixed $\sigma \in I$ the path $\left\{\varphi_{t, \sigma}\right\} \in \mathcal{P}$ is non-resonant. Then

$$
\operatorname{RFH}_{n}^{m}\left(\left\{\varphi_{t, \sigma}\right\}\right) \cong \operatorname{RFH}_{n}^{m}\left(\left\{\varphi_{t, 0}\right\}\right) \quad \forall \sigma \in I
$$

up to canonical isomorphism.

Definition 4.14. We define the set of positive contactomorphisms by

$$
\operatorname{Cont}_{0}^{+}(\Sigma):=\left\{\varphi \in \operatorname{Cont}_{0}(\Sigma) \mid \exists\left\{\varphi_{t}\right\} \in \mathcal{P} \text { with } \varphi_{1}=\varphi\right\}
$$

and define

$$
\left\{\varphi_{t}^{0}\right\} \sim\left\{\varphi_{t}^{1}\right\}
$$

if there exists a smooth family $\left\{\varphi_{t, s}\right\}_{s, t \in[0,1]}$ with $\left\{\varphi_{t, \sigma}\right\} \in \mathcal{P}$ for all $\sigma \in[0,1]$, and $\varphi_{t, 0}=\varphi_{t}^{0}$ and $\varphi_{t, 1}=\varphi_{t}^{1}$ for all $t \in[0,1]$, and $\varphi_{1, s}=\varphi_{1}^{0}=\varphi_{1}^{1} \in \operatorname{Cont}_{0}^{+}(\Sigma)$ for all $s \in[0,1]$.

Then the universal cover $\widehat{\operatorname{Cont}_{0}^{+}}(\Sigma)$ is given by -equivalence classes of paths in $\mathrm{Cont}_{0}^{+}$based at the identity. We call $\wp \in \widehat{\mathrm{Cont}_{0}^{+}}(\Sigma)$ non-resonant if one representative (and hence all representatives) are non-resonant.

REmark 4.15. By Eliashberg and Polterovich [EP00, Lemma 3.1.A] $\varphi \in$ Cont $_{0}^{+}$ if and only if the identity can be joint to $\varphi$ through a positive segment $\left\{\varphi_{t}\right\}_{t \in[0,1]}$ whose generating vector field need not be periodic.

We point out that $\operatorname{Cont}_{0}(\Sigma)$ acts on $\operatorname{Cont}_{0}^{+}(\Sigma)$ by conjugation. Indeed, if $\left\{\varphi_{t}\right\}$ is a positive path with contact Hamiltonian $h_{t}$ and $\psi \in$ Cont $_{0}$ then $\left\{\psi \varphi_{t} \psi^{-1}\right\}$ has contact Hamiltonian $\left(f h_{t}\right) \circ \psi^{-1}$ where the positive function $f$ is defined by $\psi^{*} \alpha=f \alpha$.

Moreover, discriminant points of $\left\{\varphi_{t}\right\}$ are in 1-1 correspondence with discriminant points of $\left\{\psi \varphi_{t} \psi^{-1}\right\}$ via the map $(x, \eta) \mapsto(\psi(x), \eta)$. In particular, $\left\{\varphi_{t}\right\}$ is non-resonant if and only if $\left\{\psi \varphi_{t} \psi^{-1}\right\}$ is non-resonant.

The induced action of $\psi$ on $\operatorname{Cont}_{0}^{+}(\Sigma)$ is denoted by $\mathfrak{C}_{\psi}$.

Definition 4.16. Let $\wp \in \widehat{\operatorname{Cont}_{0}^{+}}(\Sigma)$ be non-resonant. We define for integers $n, m \in$ $\mathbb{Z}$

$$
\operatorname{RFH}_{n}^{m}(\wp):=\operatorname{RFH}_{n}^{m}\left(\left\{\varphi_{t}\right\}\right)
$$

where $\left\{\varphi_{t}\right\}$ a representative of $\wp$. This is well-defined by Theorem 4.13. 
Theorem 4.13 has the following important corollary.

Corollary 4.17. Let $\wp \in \widehat{\operatorname{Cont}_{0}^{+}}(\Sigma)$ be non-resonant and let $\psi \in \operatorname{Cont}_{0}(\Sigma)$. Then for integers $n<m \in \mathbb{Z}$

$$
\operatorname{RFH}_{n}^{m}\left(\mathfrak{C}_{\psi}(\wp)\right) \cong \operatorname{RFH}_{n}^{m}(\wp)
$$

where we recall that $\mathfrak{C}_{\psi}(\wp)$ is $\wp$ conjugated by $\psi$.

Proof. Let $\psi_{s}$ be an isotopy connecting the identity and $\psi$. Then we can apply Theorem 4.13 to $\varphi_{t, s}:=\psi_{s} \varphi_{t} \psi_{s}^{-1}$ (see Remark 4.15).

\section{Diffeomorphisms of the Circle}

We consider $\Sigma=S^{1}:=\mathbb{R} / \mathbb{Z}$ with contact form $d x$. Let $a \in \mathbb{R} \backslash \mathbb{Q}$ be an irrational and positive number. Then the maps $\varphi_{t}: S^{1} \rightarrow S^{1}$ defined by

$$
\varphi_{t}(x):=x+a t
$$

is a positive and twisted period contact isotopy which is non-resonant. A pair $(x, \eta) \in$ $S^{1} \times \mathbb{R}$ is a discriminant point if and only if $\eta a \in \mathbb{Z}$. Although $\left\{\varphi_{t}\right\}$ is not non-degenerate it is of Morse-Bott type and therefore we can still define Rabinowitz Floer homology once we choose a filling. We consider two fillings of $S^{1}$.

First, we fill $S^{1}$ by the standard disk and obtain as completion $M$ the standard $M=\mathbb{R}^{2}$. In that case $S^{1}$ is Hamiltonianly displaceable thus Rabinowitz Floer homology vanishes. In fact, it holds that $\operatorname{dim} \mathrm{RFH}_{n}^{m}\left(\left\{\varphi_{t}\right\}\right) \in\{0,2\}$.

If we fill $S^{1}$ by a torus with a small disk removed we see that iterations of the Reeb orbit $\cong S^{1}$ lie all in different free homotopy classes and hence cannot be joint by a Floer differential. In particular, the complex has vanishing differential and

$$
\operatorname{dim} \operatorname{RFH}_{n}^{m}(\{\varphi\})=2\left(\left\lfloor\frac{m}{a}\right\rfloor-\left\lfloor\frac{n}{a}\right\rfloor\right) .
$$

In particular, it is possible to recover the rotation number $a$. As remarked earlier we point out that Rabinowitz Floer homology depends on the filling.

\section{A Homological Maslov Index (Boundary Value Case)}

We recall the setup. Let $(\Sigma, \xi)$ be a closed, cooriented contact manifold and $\alpha$ a fixed contact form. We assume that there exists a compact exact symplectic manifold $(\widetilde{M}, \widetilde{d})$ with $\Sigma=\partial \widetilde{M}$ and $\alpha=\left.\lambda\right|_{\Sigma}$. We attach to $\widetilde{M}$ the positive part of the symplectization of $\Sigma$, that is,

$$
M:=\widetilde{M} \cup_{\Sigma} \Sigma \times\{r \geq 1\} .
$$

On $M$ we define a 1 -form $\lambda$ by $\widetilde{\lambda}$ on $\widetilde{M}$ and $\lambda=r \alpha$ on $\Sigma \times\{r \geq 1\}$. In particular, $(M, \omega=d \lambda)$ is an exact symplectic manifold. We point out, that the entire 
symplectization $S \Sigma$ of $\Sigma$ embeds into $M$ via the flow of the Liouville vector field of $\lambda$.

In addition we assume that we are given two Lagrangian submanifolds $L_{0}, L_{1}$ inside $M$ with the following properties for $i=0,1$ :

- $\left.\lambda\right|_{L_{i}}=0$ and $L_{i} \pitchfork \Sigma=: \Lambda_{i}$ is a closed Legendrian submanifold and

- $L_{i} \cap(\Sigma \times\{r \geq 1\})=\Lambda_{i} \times\{r \geq 1\}$.

An example is given by $M=T^{*} B, \Sigma=S^{*} B$, a unit cotangent bundle, and $L_{i}=T_{q_{i}}^{*} B$ for $q_{i} \in B$. We define the path space

$$
\mathscr{P}:=\left\{u:[0,1] \rightarrow M \mid u(i) \in L_{i}, i=0,1\right\} .
$$

For a function

$$
F: M \times S^{1} \rightarrow \mathbb{R}
$$

we define the Rabinowitz action functional

$$
\mathcal{A}: \mathscr{P} \times \mathbb{R} \rightarrow \mathbb{R}
$$

by precisely the same formula as above (see (3.1)). Since $\left.\lambda\right|_{L_{i}}=0$ the critical point equation of $\mathcal{A}$ is unchanged. Thus, a pair $(u, \eta) \in \mathscr{P} \times \mathbb{R}$ is a critical point of $\mathcal{A}$ if and only if the following equations hold

$$
\left.\begin{array}{l}
\dot{u}(t)=\eta X_{F_{\eta t}}(u(t)) \\
F_{\eta}(u(1))=0
\end{array}\right\} .
$$

Again following Givental [Giv89, Giv90a, Giv90b] we make the following definition.

Definition 6.1. Let $\left\{\varphi_{t}\right\}$ be a smooth path in $\operatorname{Cont}(\Sigma)$. Then a pair $(x, \eta) \in \Lambda_{0} \times \mathbb{R}$ is called a Legendrian discriminant point (with respect to $\left\{\varphi_{t}\right\}$ ) if

$$
\varphi_{\eta}(x) \in \Lambda_{1} .
$$

Using Proposition 2.3 we assign to the path $\left\{\varphi_{t}\right\}$ the contact Hamiltonian $H_{t}$ : $S \Sigma \rightarrow \mathbb{R}$. If we set

$$
F_{t}(x, r):=H_{t}(x, r)-1
$$

then the critical points of $\mathcal{A}$ are again in 1-1 correspondence with Legendrian discriminant points. For a positive and twisted path $\left\{\varphi_{t}\right\} \in \mathcal{P}(\Sigma, \xi)$ we define as in Definition 4.9

$$
\operatorname{RFH}_{a}^{b}\left(\left\{\varphi_{t}\right\} ; L_{0}, L_{1}\right) .
$$

We refer the reader to [Mer10] for details on Lagrangian Rabinowitz Floer homology. 


\section{Asymptotics and Obstructions to Positive Loops in $\operatorname{Cont}(\Sigma)$}

We assume the same setting as in Section 6. We fix an element $\left\{\varphi_{t}\right\} \in \mathcal{P}$ and consider the maps induced by inclusion

$$
i^{n, m}: \operatorname{RFH}_{0}^{m}\left(\left\{\varphi_{t}\right\}\right) \rightarrow \operatorname{RFH}_{0}^{n}\left(\left\{\varphi_{t}\right\}\right) .
$$

Then the sequence $n \mapsto \operatorname{dim}\left(\operatorname{im} i^{n, m}\right)$ is non-increasing and we set

$$
\mu(m):=\min _{n}\left\{\operatorname{dim}\left(\operatorname{im} i^{n, m}\right)\right\} .
$$

The map $m \mapsto \mu(m)$ is non-decreasing and we consider the growth rate of $m \mapsto \mu(m)$. The following is second Theorem from the Introduction.

Theorem 7.1. Let $B$ be a closed manifold with finite fundamental group such that the rational cohomology ring has at least two generators. Then $\Sigma:=S^{*} B$ with its standard contact structure $\xi$ admits no closed positive loops in $\operatorname{Cont}(\Sigma, \xi)$.

REMARK 7.2. Theorem 7.1 can be seen as a complement to a result by Chernov-Nemirovski. Indeed, if the fundamental group of the manifold $B$ is infinite then there exist no positive loops in $\operatorname{Cont}\left(S^{*} B\right)$ (see [CN10, Corollary 8.1]).

According to Eliashberg et al. [EKP06] there are never positive contractible loops of contactomorphism of $S^{*} B$ since $S^{*} B$ is orderable. Strictly speaking EliashbergKim-Polterovich could not treat the case of a manifold whose fundamental group is infinite but has only finitely many conjugacy classes. The general case is covered by the aforementioned result by Chernov-Nemirovski.

If the fundamental group is finite but the rational cohomology ring is generated by only one element there exist examples of positive loops in $\operatorname{Cont}\left(S^{*} B\right)$. For instance the geodesic flow of any P-metric, e.g. the round metric on $B=S^{n}$, gives rise to such a positive loop (see [Bes78]). Of course, these loops are not contractible by the result of Eliashberg-Kim-Polterovich.

Proof of Theorem 7.1. We argue by contradiction. Let $\left\{\varphi_{t}\right\}$ be a positive loop in $\operatorname{Cont}(\Sigma, \xi)$. In particular, it is twisted periodic: $\left\{\varphi_{t}\right\} \in \mathcal{P}$. As above we set $L_{i}:=T_{q_{i}}^{*} B$ for $q_{i} \in B$. Then for generic $q_{0}, q_{1} \in B$ the corresponding Rabinowitz action functional is Morse and its Rabinowitz Floer homology

$$
\operatorname{RFH}_{a}^{b}\left(\left\{\varphi_{t}\right\} ; L_{0}, L_{1}\right)
$$

is well defined (see [Mer10]). Since $\left\{\varphi_{t}\right\}$ is a loop the number of critical points of the underlying Rabinowitz action functional grows linearly with the action value. Therefore, the growth rate of the function $m \mapsto \mu(m)$ is at most linear.

As in Definition 2.1 we assign the 1-periodic, positive function $h_{t}: \Sigma \rightarrow \mathbb{R}_{>0}$ to $\left\{\varphi_{t}\right\}$. We can homotope $h_{t}$ through positive and 1-periodic functions to the function

$$
k(q, p):=\frac{1}{2}|p|_{g}^{2}
$$


where $g$ is a bumpy metric on $B$. The contact flow $\left\{\psi_{t}\right\}$ induced by $k$ is just the geodesic flow on $S_{g}^{*} B$ associated to $g$. Arguing as in [AF10, Section 5] it follows that the growth rate of the positive, twisted periodic path $\left\{\psi_{t}\right\}$ coincides with the growth rate of $\left\{\varphi_{t}\right\}$. In particular, the growth rate of $\left\{\psi_{t}\right\}$ is at most linear.

According to [Mer10, Theorem B] the Rabinowitz Floer homology in positive degrees of the path $\left\{\psi_{t}\right\}$ is isomorphic to the homology of the based loop space. It follows from Gromov's theorem [Gro78, Gro07] (see also [Pat99]) that if the homology of the loop space grows at most linearly in action then it also grows at most linearly in degree. Using the theory of minimal models by Sullivan [Sul75] and arguing as in the proof of the Bott-Samelson theorem in [Bes78, Chapter 7.D] it follows that the based loop space of a closed manifold with finite fundamental group such that the rational cohomology ring has at least two generators grows at least quadratically.

This contradiction finishes the proof of the theorem.

\section{Acknowledgments}

This article was written during a visit of the authors at the Forschungsinstitut für Mathematik (FIM), ETH Zürich. The authors thank the FIM for its stimulating working atmosphere. The present article originates from inspiring discussions with Leonid Polterovich. The authors express their gratitude. This material is supported by the National Science Foundation Grant DMS-0903856 (PA) and by the Basic Research fund 2010-0007669 funded by the Korean government (UF).

\section{References}

[AF10] P. Albers and U. Frauenfelder. Spectral invariants in Rabinowitz-Floer homology and global Hamiltonian perturbations. Journal of Modern Dynamics, (2)4 (2010), 329-357.

[Bes78] A.L. Besse. Manifolds All of Whose Geodesics are Closed, Ergebnisse der Mathematik und ihrer Grenzgebiete, Vol. 93. Springer-Verlag, Berlin (1978).

[BS07] G. Ben Simon. The nonlinear Maslov index and the Calabi homomorphism. Communications in Contemporary Mathematics, (6)9 (2007), 769-780.

[CF09] K. Cieliebak and U. Frauenfelder. A Floer homology for exact contact embeddings. Pacific Journal of Mathematics, (2)293 (2009), 251-316.

[CFO10] K. Cieliebak, U. Frauenfelder, and A. Oancea. Rabinowitz Floer homology and symplectic homology. Ann. Sci. Éc. Norm. Supér. (4), (6)43 (2010), $957-1015$.

[CN10] V. Chernov and S. Nemirovski. Non-negative Legendrian isotopy in $S T^{*} M$. Geometry \& Topology, (1)14 (2010), 611-626.

[EKP06] Y. Eliashberg, S. Kim, and L. Polterovich. Geometry of contact transformations and domains: orderability versus squeezing. Geometry \& Topology, 10 (2006), 1635-1747 (electronic).

[EP00] Y. Eliashberg and L. Polterovich. Partially ordered groups and geometry of contact transformations. Geometric and Functional Analysis, (6)10 (2000), 14481476. 
[EP03] M. Entov and L. Polterovich. Calabi quasimorphism and quantum homology. International Mathematics Research Notices, (30) (2003), 1635-1676.

[Giv89] A.B. Givental. Periodic mappings in symplectic topology. Funktsional. Anal. $i$ Prilozhen., (4)23 (1989), 37-52, 96.

[Giv90a] A.B. Givental. Nonlinear Generalization of the Maslov Index, Theory of singularities and its applications, Adv. Soviet Math., Vol. 1. Amer. Math. Soc., Providence, RI (1990), pp. 71-103.

[Giv90b] A.B. Givental. The Nonlinear Maslov Index, Geometry of low-dimensional manifolds, 2 (Durham, 1989), London Math. Soc. Lecture Note Ser., Vol. 151. Cambridge Univ. Press, Cambridge (1990), pp. 35-43.

[Gro78] M. Gromov. Homotopical effects of dilatation. Journal of. Differential Geometry, (3)13 (1978), 303-310.

[Gro07] M. Gromov. Metric Structures for Riemannian and Non-Riemannian Spaces, English ed. Modern Birkhäuser Classics, Birkhäuser Boston Inc., Boston, MA (2007).

[Mer10] W. MERRY, Lagrangian Rabinowitz Floer homology and twisted cotangent bundles (2010), arXiv:1010.4190.

[Pat99] G. Paternain, Geodesic Flows, Progress in Mathematics, Vol. 180. Birkhäuser Boston Inc., Boston, MA (1999).

[Sul75] D. Sullivan, Differential Forms and the Topology of Manifolds, ManifoldsTokyo 1973 (Proc. Internat. Conf., Tokyo, 1973). Univ. Tokyo Press, Tokyo (1975), pp. 37-49.

Peter Albers, Department of Mathematics, Purdue University, West Lafayette, USA palbers@math.purdue.edu

Urs Frauenfelder, Department of Mathematics, Research Institute of Mathematics, Seoul National University, Seoul, Korea frauenf@snu.ac.kr 